\title{
Estratificación de riesgo para enfermedad coronaria en adultos mayores
}

\author{
Carolina Pemberthy-López ${ }^{\mathrm{a}, \mathrm{g}, *}$, Nicolás Jaramillo-Gómez ${ }^{\mathrm{b}, \mathrm{g}}$, \\ Jonathan Cardona-Vélez ${ }^{\mathrm{c}, \mathrm{g}}$, Camilo Andrés Velásquez-Mejía ${ }^{\mathrm{d}, \mathrm{g}}$, \\ Valentina Jaramillo-Restrepo ${ }^{\mathrm{e}, \mathrm{g}}$ y Heidy Contreras-Martínez ${ }^{\mathrm{f}, \mathrm{g}}$
}

\author{
a Departamento de Medicina Interna, Universidad de Antioquia, Medellín, Colombia \\ b Departamento de Medicina Interna, Universidad CES, Universidad de Córdoba, Medellín, Colombia \\ c Departamento de Medicina Interna, Universidad Pontificia Bolivariana, Medellín, Colombia \\ d Departamento de Medicina Interna, Universidad CES, Medellín, Colombia \\ e Facultad de Medicina, Universidad CES, Medellín, Colombia \\ f Universidad de Antioquia, Universidad CES, Medellín, Colombia \\ g Cardiovital, CEMDE, Universidad CES, Medellín, Colombia
}

Recibido el 19 de octubre de 2015; aceptado el 28 de diciembre de 2015

Disponible en Internet el 3 de marzo de 2016

\section{PALABRAS CLAVE \\ Enfermedad \\ coronaria; \\ Anciano; \\ Edad}

\section{KEYWORDS}

Coronary disease;

Elderly;

Age

\begin{abstract}
Resumen La población de adultos mayores ha tenido muy poca representación en los diferentes estudios clínicos, afectando el conocimiento sobre el tratamiento adecuado de este grupo etario. Las determinaciones de excluir a este tipo de población de dichos estudios se hacen basadas en un incremento en la probabilidad de presentar mayores eventos adversos, aumentando la complejidad del análisis de los resultados.

Se hace necesario utilizar escalas válidas que permitan evaluar el verdadero riesgo de estos pacientes para desarrollar complicaciones secundarias a procedimientos invasivos. Escalas como: CRUSADE, Italian Elderly ACS, THE STORM y el Consenso Francés, demuestran que al estratificar estos pacientes se logra de forma objetiva determinar la población de adultos mayores que se benefician de una intervención intensiva para lograr mejores desenlaces.

( $) 2016$ Sociedad Colombiana de Cardiología y Cirugía Cardiovascular. Publicado por Elsevier España, S.L.U. Este es un artículo Open Access bajo la licencia CC BY-NC-ND (http:// creativecommons.org/s/by-nc-nd/4.0/).
\end{abstract}

Risk stratification for coronary disease in old adults

Abstract Old adult population has suffered from under-representation in different clinical studies, thus affecting knowledge regarding best treatments for this age group. Reasons to

\footnotetext{
* Autor para correspondencia.

Correo electrónico: caropember@gmail.com (C. Pemberthy-López).
} 
exclude this type of patients in those studies are based on the increased likelihood of presenting more adverse effects, thus raising the complexity of results analysis.

It is necessary to use valid scales that can allow to assess the real risk for these patients to develop complications secondary to invasive procedures. Scales such as: CRUSADE, Italian Elderly ACS, THE STORM and French Consensus reveal that stratification of these patients allows for and objective determination of the population of old adults that benefit from an intensive surgery to improve outcomes.

(c) 2016 Sociedad Colombiana de Cardiología y Cirugía Cardiovascular. Published by Elsevier España, S.L.U. This is an open access article under the CC BY-NC-ND license (http:// creativecommons.org/licenses/by-nc-nd/4.0/).

\section{Introducción}

La edad como determinante de la vida, asociado a diferentes comorbilidades constituye un reto para el médico y los sistemas de salud. La tendencia de nuestro sistema actual de salud de evaluar y clasificar los pacientes ancianos, solo por su edad cronológica, crea una barrera que se torna en un impedimento para generar una adecuada conducta, incluso genera un dilema ético difícil de resolver para el médico, cuando no se le dan los elementos de análisis suficientes para encarar este tipo de pacientes, que dada las tendencias demográficas actuales, está en franco aumento. La decisión de realizar una intervención específica, sea diagnóstica o terapéutica, en los pacientes de edad, no es fácil de determinar, ya que cualquier insulto físico puede romper la homeostasis del organismo en pacientes aparentemente saludables y desencadenar procesos que pueden terminar en la muerte; es en este grupo etario, en donde la respuesta a una intervención determinada presenta una gran variedad. Las intervenciones intensivas pueden generar mejores incidencias en los desenlaces que en poblaciones más jóvenes. Por esto, se debe determinar la fragilidad del individuo, la cual se define como la incapacidad para adaptarse a situaciones estresantes tanto internas como externas, manifestándose como una descompensación al enfrentarse a condiciones patológicas que aumentan el riesgo de eventos adversos. Para guiar de esta manera el manejo, desafortunadamente, no existe una escala universal validada para determinar qué paciente se beneficia de un procedimiento invasivo versus un manejo médico conservador, y los criterios clínicos se vuelven la herramienta más importante para tomar esta decisión. Criterios como: comorbilidades y su control, soporte social, funcionalidad, estado cognitivo, fragilidad y voluntad del paciente determinarán el accionar ${ }^{1}$. Fried y Col., establecen criterios para determinar la fragilidad en adultos mayores, definiendo un fenotipo que permite referirse a un estado de prefragilidad y fragilidad establecida. No obstante, deja a un lado factores que desempeñan un papel importante en dicho estado ${ }^{2}$. Esto limita su interpretación, aunque lo hace más amigable para el uso diario.

Surgen escalas que utilizan mayor número de criterios clínicos de forma que puedan ser aplicados a pacientes con enfermedades cardiacas y de esta manera determinar el riesgo y los posibles desenlaces. Múltiples guías recomiendan realizar una estratificación de riesgo temprana usando sistemas validados como la escala de GRACE (Global Registry of Acute Coronary Events), para identificar apropiadamente los candidatos a terapia invasiva. La edad es un componente importante de la estratificación, considerando que la mortalidad intrahospitalaria es significativamente alta en los adultos mayores. Sin embargo, se ha encontrado que estas escalas de estratificación no son tan usadas como sí lo es la evaluación subjetiva del riesgo de un paciente ${ }^{3}$. Estudios como el MINAP (Myocardial Ischemia National Audit Project), demuestran que en los adultos mayores no hay una adecuada estratificación, llevando a un manejo inapropiado ${ }^{4}$. Evidencia del registro canadiense ACS 2 sugiere que la complejidad de las escalas las hace poco prácticas en el manejo diario del médico, con una pobre correlación entre la evaluación de riesgo hecha por él y las escalas validadas ${ }^{5}$.

Se han propuesto algunas escalas de estratificación específicas para adultos mayores que tratan de proporcionar herramientas al médico para enfrentarse a la patología cardiovascular en este grupo etario. Revisando la evidencia científica se encuentran escalas específicas tales como: la escala de riesgo CRUSADE (Can Rapid Stratification of unstable Angina Patients Supress Adverse Outcomes with Early Implementation of the American College of Cardiology/American heart Association Guidelines), la escala del estudio Italian Elderly ACS, la escala del estudio THE STORM. Adicionalmente, se cuenta con los modelos predictores y las escalas como la presentada en el Consenso Francés, las cuales proporcionan variables necesarias para una evaluación global y determinar un manejo apropiado.

\section{Escalas de riesgo en adultos mayores}

\section{Escala CRUSADE}

En el año 2011 se publicó una escala realizada a partir del registro $\mathrm{CRUSADE}^{6}$, que permitió establecer un modelo de predicción de mortalidad a 1 año; se tomaron 43.239 pacientes con infarto agudo de miocardio sin elevación del ST (IAMNST) y edad $\geq 65$ años entre los años 2003-2006 y se cruzaron con los datos del Centers for Medicare and Medicaid Services para definir la mortalidad por todas las causas, con una media de seguimiento de 453 días. La población se dividió en dos subgrupos: la cohorte de derivación $(n=34.640$, $80 \%$ del total) y cohorte de validación $(n=8599,20 \%$ del 
Tabla 1 Escala de riesgo y nomograma CRUSADE

\begin{tabular}{|c|c|c|}
\hline Variable & Descripción & Puntuación \\
\hline \multirow[t]{5}{*}{ Edad (años) } & $\leq 70$ & 0 \\
\hline & $\overline{71-75}$ & 5 \\
\hline & $76-80$ & 10 \\
\hline & $81-85$ & 14 \\
\hline & $\geq 86$ & 19 \\
\hline \multirow{4}{*}{$\begin{array}{l}\text { Creatinina sérica inicial } \\
\qquad(\mathrm{mg} / \mathrm{dl})+\end{array}$} & $\leq 1$ & 0 \\
\hline & $\overline{1}-1.99$ & 5 \\
\hline & $2-2.99$ & 11 \\
\hline & $\geq 3$ & 16 \\
\hline \multirow{3}{*}{$\begin{array}{l}\text { Presión arterial sistólica } \\
\text { inicial }(\mathrm{mmHg})\end{array}$} & $\leq 90$ & 2 \\
\hline & $91-100$ & 1 \\
\hline & $>100$ & 0 \\
\hline \multirow{2}{*}{$\begin{array}{l}\text { Signos de falla cardiaca } \\
\text { al ingreso }\end{array}$} & No & 0 \\
\hline & $\mathrm{Si}$ & 10 \\
\hline \multirow{4}{*}{$\begin{array}{l}\text { Frecuencia cardiaca inicial } \\
\text { (latidos/minuto) }\end{array}$} & $<90$ & 0 \\
\hline & $90-99$ & 2 \\
\hline & $100-109$ & 3 \\
\hline & $\geq 100$ & 5 \\
\hline \multirow[t]{3}{*}{ Peso (Kg) } & $\leq 60$ & 10 \\
\hline & $61-80$ & 5 \\
\hline & $\geq 81$ & 0 \\
\hline \multirow{2}{*}{$\begin{array}{l}\text { Antecedente de falla } \\
\text { cardiaca }\end{array}$} & No & 0 \\
\hline & $\mathrm{Si}$ & 8 \\
\hline \multirow[t]{3}{*}{ Hematocrito inicial (\%) } & $<30$ & 5 \\
\hline & $30-39.9$ & 3 \\
\hline & $\geq 40$ & 0 \\
\hline \multirow{4}{*}{$\begin{array}{l}\text { Relación troponina } \\
\text { inicial }(x \text { LSN)* }\end{array}$} & $\leq 1$ & 0 \\
\hline & $1.1-3$ & 1 \\
\hline & $3.1-5$ & 3 \\
\hline & $\geq 5.1$ & 5 \\
\hline \multirow[t]{2}{*}{ Antecedente de ACV } & $\overline{\text { No }}$ & 0 \\
\hline & $\mathrm{Si}$ & 6 \\
\hline \multirow[t]{2}{*}{ Diabetes mellitus } & No & 0 \\
\hline & $\mathrm{Si}$ & 4 \\
\hline \multirow[t]{2}{*}{ Sexo } & Femenino & 0 \\
\hline & Masculino & 4 \\
\hline \multirow{2}{*}{$\begin{array}{l}\text { Antecedente de enfermedad } \\
\text { arterial periférica }\end{array}$} & No & 0 \\
\hline & $\mathrm{Si}$ & 3 \\
\hline
\end{tabular}

+Pacientes en diálisis tienen puntuación de 16.

*Relación troponina inicial: valor troponina inicial/límite superior de normalidad de troponina de laboratorio local.

total). De todas las variables relacionadas con la mortalidad a 1 año, se tomaron en cuenta para la construcción de la escala 13 variables; aquellas con mayor significancia clínica y estadística, con capacidad de discriminación comparables entre la población de derivación y la de validación (c- estadístico 0.754 y 0.744 respectivamente) y con buena calibración a través del espectro de riesgo. Después de establecer la puntuación de riesgo según la suma de los valores establecidos, se evalúa en un nomograma su relación con la mortalidad a 1 año, ver tabla 1 y Fig. 1.

La mediana de la escala de riesgo en la cohorte de derivación fue 32 puntos (percentil $25-75=21$ - 43 puntos), con un evidente incremento en la mortalidad a 1 año a medida que la categoría de riesgo aumenta, ver Fig. 2.

Esta escala de riesgo permite evaluar desenlaces a largo plazo basado en las variables tomadas al momento del

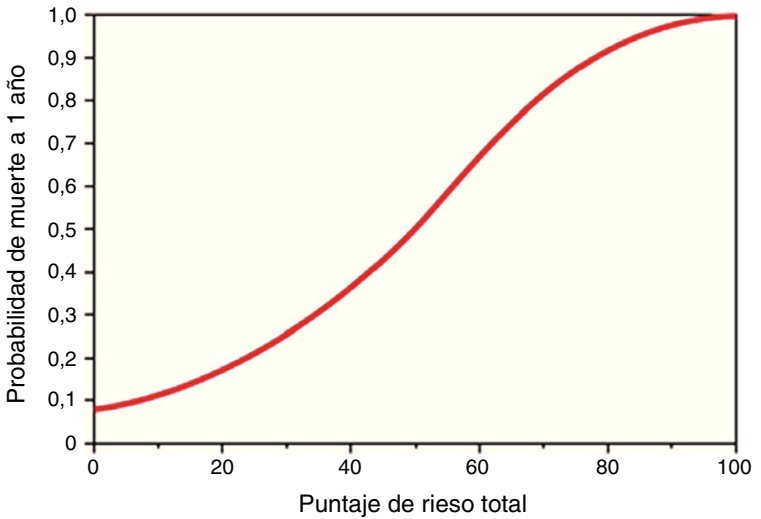

Figura 1 Nomograma del registro CRUSADE. Tomado de Roe et al. ${ }^{6}$.

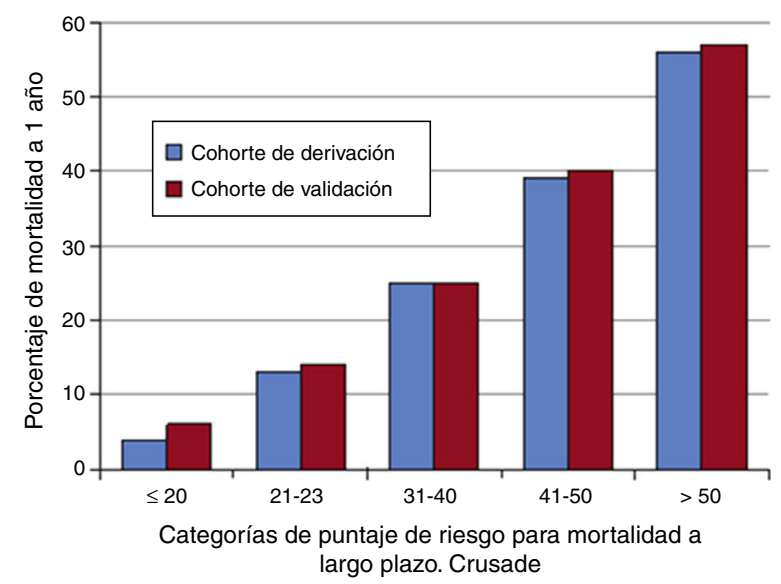

Figura 2 Porcentaje de las categorías de mortalidad a largo plazo. Tomado de Roe et al. ${ }^{6}$.

ingreso hospitalario, facilitando su aplicación. No obstante, no tiene en cuenta otros factores que influyen en la mortalidad como la malignidad, otras comorbilidades asociadas y la fragilidad. Al momento de la realización de la presente revisión no se encontró en la literatura otras validaciones de esta escala de riesgo.

\section{Estudio Italian Elderly ACS}

El estudio Italian Elderly $\mathrm{ACS}^{7}$ ofreció una alternativa para la estratificación de riesgo en pacientes con IAMNST y edad $\geq 75$ años, desarrollando y validando una escala de predicción del riesgo de la mortalidad por todas las causas a 1 año. Se evaluaron 313 pacientes, durante un tiempo promedio de 350 días; analizando las características al momento del ingreso hospitalario e incluyendo en la escala aquellas que tuvieron asociación independiente y significativa con la mortalidad. Se estableció una escala de cinco variables con una fuerte capacidad discriminatoria (AUC 0.739; IC 95\%=0.686$0.787)$.

Se clasificaron en 3 categorías: bajo riesgo: $\leq 1$ punto; riesgo moderado: 2 puntos; alto riesgo: $\geq 3$ puntos. Posteriormente se validó la escala en una cohorte de 332 pacientes, con una alta capacidad de discriminación (AUC 0.700; IC 95\%=0.631-0.758); la Fig. 3 muestra la 

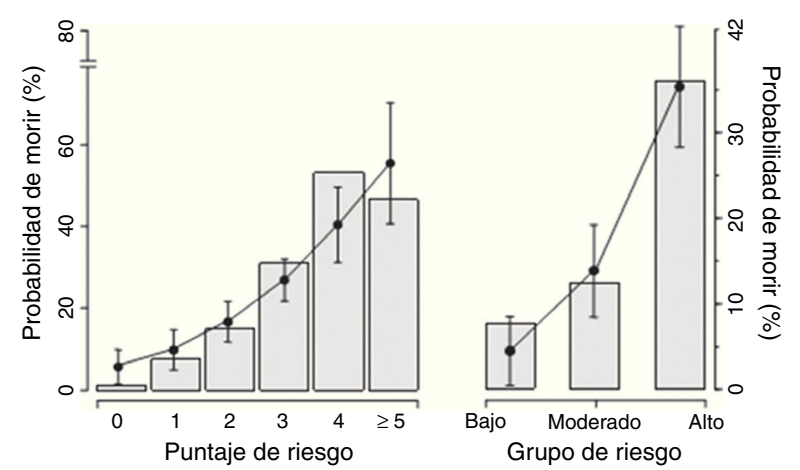

Figura 3 Riesgo de mortalidad por todas las causas. Estudio Italian Elderly ACS. Riesgo de mortalidad por todas las causas observada (barras) y predicha (círculos) en la cohorte de validación de acuerdo al puntaje de riesgo (izquierda). Las líneas sólidas a través de cada barra representan 95\% IC del riesgo predicho. El análisis es replicado (derecha) de acuerdo a la estratification del riesgo (bajo rieso $\leq 1$; moderado $=2$, alto rieso $\geq 3$ ) derivada de la cohote del estudio. Tomado de Angeli et al. ${ }^{7}$.

Tabla 2 Escala del estudio Italian Elderly ACS

\begin{tabular}{|c|c|c|}
\hline Variable & Puntuación & \\
\hline Hemoglobina $<10 \mathrm{~g} / \mathrm{dl}$ & 2 & Puntaje mínimo $=0$ \\
\hline $\begin{array}{l}\text { Niveles de troponinas } \\
\text { elevados }\end{array}$ & 1 & Puntaje máximo $=6$ \\
\hline $\begin{array}{l}\text { ECG con cambios } \\
\text { isquémicos }\end{array}$ & 1 & Mortalidad: \\
\hline $\begin{array}{c}\mathrm{TFG}<45 \mathrm{ml} / \mathrm{min} / \\
1.73 \mathrm{~m}^{2} £\end{array}$ & 1 & $2 \%=0$ puntos \\
\hline $\begin{array}{l}\text { Evento vascular } \\
\text { previo }\end{array}$ & 1 & $75 \%=6$ puntos \\
\hline
\end{tabular}

correlación entre la estratificación de riesgo y la probabilidad de muerte.

La presente escala permite realizar una estratificación del riesgo basado en variables que se pueden obtener al momento del ingreso hospitalario, es fácil de aplicar y su capacidad predictiva fue consistente en ambos géneros y en los diferentes grupos de edades. Sin embargo, se excluyeron pacientes con patologías que pueden influir en la mortalidad como: malignidad, antecedente de accidente cerebrovascular (ACV), fragilidad, déficit neurológico, enfermedad pulmonar obstructiva crónica (EPOC) severa, falla cardiaca refractaria a tratamiento, creatinina $>2.5 \mathrm{mg} / \mathrm{dl}$. Todo lo cual limita su aplicación en este grupo poblacional (tabla 2).

\section{Estudio THE STORM}

El estudio THE STORM (Acute Coronary Syndrome in Patients end of Life and Risk Assesment) ${ }^{8}$, evaluó el papel de las escalas de estratificación clásicas frente a los criterios 'Gold Standards Framework Prognostic Indicator Guide' (GSF), establecidos inicialmente para pacientes con cáncer, pero que ha demostrado ser útil en la valoración de los pacientes
Tabla 3 Criterios evaluados en el estudio THE STORM

CRITERIOS GENERALES
1. Pérdida de peso $>10 \%$ en los últimos 6 meses.
2. Declinación física general.
3. Albúmina sérica $<25 \mathrm{~g} / \mathrm{l}$.
4. Reducción del nivel de desempeño (Karnofsky $<50 \%$ ).
CRITERIOS ESPECÍFICOS DE ENFERMEDAD CARDIACA
1. La pregunta sorpresa (preguntar a un profesional de la
salud familiarizado con el paciente): ¿Se sorprendería si el
paciente muere en los próximos $6-12$ meses?
2. Falla cardiaca NYHA (New York Heart Association) III o Iv.
3. Hospitalizaciones repetitivas en el último año.
4. Síntomas físicos o psicológicos persistentes a pesar
de terapia óptima tolerada.

con múltiples comorbilidades no oncológicas, permitiendo identificar aquellos que se acercan al final de la vida y que se podrían beneficiar de manejo paliativo/conservador. Se evaluaron los criterios (tabla 3), al momento del ingreso y se consideró GSF positivo si cumplía 1 criterio general y 2 criterios de enfermedad cardiaca.

El GSF positivo se asoció con mayores tasas de eventos no cardiovasculares a 3 meses $(22.7 \%$ vs. $6.7 \%$; $p=0.03$ ) y mayores tasas de eventos cardiovasculares y no cardiovasculares a 12 meses ( $36 \%$ vs. $16.4 \% ; p=0.04$ y $27.3 \%$ vs. $6.7 \% ; p=0.009$, respectivamente). El score de GRACE fue un predictor de eventos cardiovasculares, mientras que el GSF positivo fue predictor independiente de eventos no cardiovasculares; por tanto, se podrían usar ambos scores a la hora de elegir el tratamiento apropiado. Sin embargo, se requiere validación externa para extender el uso de esta estrategia.

\section{Otras escalas}

El estudio realizado por Maraschini A. y Col., demostró que el modelo Italian Coronary Artery Bypass Graft Outcome Project (ItCABG) y el modelo logístico European System for Cardiac Operative Risk Evaluation (LogEuroSCORE), fueron pobres predictores de mortalidad para pacientes octogenarios y concluyen que deben ser evaluados los factores de riesgo específicos con el fin de mejorar la estratificación del riesgo en pacientes con una edad igual o mayor a 80 años $^{9}$.

\section{Consenso francés}

El Consenso de la Sociedad Francesa de Gerontología y Geriatría y la Sociedad Francesa de Cardiología para el manejo de la enfermedad coronaria en adultos mayores, sugiere realizar una evaluación geriátrica global para tomar decisiones de manejo, dentro de la cual se evalúa: la función cognitiva, la dependencia para la realización de actividades diarias, los trastornos de la marcha, el riesgo de caídas, el estado nutricional, la presencia de depresión, el soporte social y el acceso a los servicios de salud ${ }^{10}$.

El Mini Mental State Examination (MMS) es una prueba simple, rápida y estandarizada para determinar la función cognitiva. El valor umbral depende de la edad del paciente y su nivel sociocultural. Una puntuación de más de 27 se 
considera normal, por debajo de 24 es anormal y señala la necesidad de una evaluación detallada de la función cognitiva en una unidad especializada. Una puntuación entre 24 y 27 se considera anormal en pacientes menores de 80 años, con un alto nivel sociocultural y con trastornos de la memoria $u$ otras funciones cognitivas ${ }^{11,12}$. La valoración de la dependencia para la realización de actividades diarias puede hacerse mediante la escala ADL (Activities of Daily Living Score) o IADL (Instrumental Activities of Daily Living Score), donde se le pregunta al paciente y sus contactos cercanos acerca de: la higiene personal, el uso del sanitario, la continencia urinaria e intestinal y la asistencia para vestirse, comer o movilizarse. El sujeto es considerado como dependiente, cuando necesita ayuda para llevar a cabo la actividad correspondiente ${ }^{13}$.

La evaluación geriátrica somática tiene en cuenta la estimación de los trastornos de la marcha y el riesgo de caídas a través de la prueba de postura unipedal, con el objeto de valorar la capacidad de pararse en una pierna durante 5 segundos. Del mismo modo, el estado nutricional a través de las escalas validadas (escala MNA) para el análisis de la albúmina o prealbúmina plasmáticas. Para determinar la presencia de depresión, se propone la Geriatric Depression Scale (GDS) simplificada, la cual ofrece una evaluación inicial del estado de ánimo en estos pacientes, pues la depresión es muy frecuente en los adultos mayores y puede afectar tanto las manifestaciones de la enfermedad coronaria como su tratamiento. Además, representa un factor de riesgo independiente para la mortalidad cardiovascular; por tanto, su detección y manejo son fundamentales en esta población ${ }^{14}$.

Otros parámetros igualmente importantes son: la evaluación de las condiciones de vida y de la gestión de la salud comunitaria, ya que permiten estimar la adherencia al tratamiento propuesto, evaluar el acceso a los servicios de salud y evaluar su condición social así como el rol de los cuidadores (relaciones familiares o amigos). En términos generales, la estrategia terapéutica no se basa en la edad real del paciente, sino más bien en un análisis individual, teniendo en cuenta la severidad de la enfermedad coronaria, comorbilidades, esperanza y calidad de vida del paciente $^{15}$.

\section{Fragilidad}

La fragilidad se caracteriza por la vulnerabilidad a estresores agudos, como consecuencia de la declinación funcional y de las reservas fisiológicas. Incluye dominios como: la movilidad, la fuerza, el equilibrio, el procesamiento motor, la cognición, la nutrición, la resistencia y la actividad física ${ }^{16}$. Su evaluación representa una medida de la edad biológica y permite predecir eventos adversos en la población anciana. Se ha demostrado que la fragilidad predice complicaciones postoperatorias y mortalidad en pacientes llevados a cirugía cardiaca y se correlaciona con la calidad de vida, las hospitalizaciones, y la mortalidad independiente de la edad, el género y las comorbilidades. Existen varias escalas para evaluar la fragilidad, no obstante, solo algunas se han estudiado en pacientes con la enfermedad coronaria.

Murali-Krishnan y Col. realizaron un estudio con 745 pacientes que ingresaron para coronariografía con el fin de
Tabla 4 Escala del Canadian Study of Health and Aging Clinical Frailty

\begin{tabular}{|c|c|}
\hline 1. Muy saludable & $\begin{array}{l}\text { Personas activas, energéticas y } \\
\text { motivadas. Usualmente se ejercitan } \\
\text { de forma regular. }\end{array}$ \\
\hline $\begin{array}{l}\text { 2. Buen estado } \\
\text { de salud }\end{array}$ & $\begin{array}{l}\text { Personas sin síntomas activos de } \\
\text { enfermedad pero son menos } \\
\text { saludables que los de categoría 1. A } \\
\text { menudo ejercitan o son muy activos } \\
\text { ocasionalmente. Ejemplo: } \\
\text { por temporadas. }\end{array}$ \\
\hline $\begin{array}{l}\text { 3. Adecuado } \\
\text { estado de salud }\end{array}$ & $\begin{array}{l}\text { Personas cuyos problemas médicos } \\
\text { están bien controlados, no tienen una } \\
\text { actividad regular más allá de las } \\
\text { caminatas rutinarias. }\end{array}$ \\
\hline 4. Vulnerable & $\begin{array}{l}\text { No son dependientes para las } \\
\text { actividades diarias, pero } \\
\text { frecuentemente los síntomas limitan } \\
\text { las actividades. Una queja común es } \\
\text { estar "lento" y/o lento durante el } \\
\text { día. }\end{array}$ \\
\hline 5. Levemente frágil & $\begin{array}{l}\text { Tienen un enlentecimiento más } \\
\text { evidente y necesitan ayuda en } \\
\text { actividades diarias avanzadas } \\
\text { (finanzas, transporte, } \\
\text { medicamentos). Progresivamente } \\
\text { limita la realización de compras y } \\
\text { salir a caminar solo, preparación de } \\
\text { las comidas y actividades del hogar. }\end{array}$ \\
\hline $\begin{array}{l}\text { 6. Moderadamente } \\
\text { frágil }\end{array}$ & $\begin{array}{l}\text { Necesitan ayuda con todas las } \\
\text { actividades fuera y dentro de la casa. } \\
\text { Tienen problemas con las escaleras } \\
\text { y necesitan ayuda para el baño. }\end{array}$ \\
\hline $\begin{array}{l}\text { 7. Severamente } \\
\text { frágil }\end{array}$ & $\begin{array}{l}\text { Completamente dependiente para el } \\
\text { cuidado personal por cualquier causa } \\
\text { (física o cognitiva). A pesar de ello, } \\
\text { parecen estables y no en alto riesgo } \\
\text { de morir (en los próximos } 6 \text { meses). }\end{array}$ \\
\hline $\begin{array}{l}\text { 8. Marcadamente } \\
\text { frágil }\end{array}$ & $\begin{array}{l}\text { Completamente dependiente, } \\
\text { aproximándose al final de la vida. } \\
\text { Típicamente incapaces de } \\
\text { recuperarse incluso de enfermedad } \\
\text { leve. }\end{array}$ \\
\hline $\begin{array}{l}\text { 9. Enfermo } \\
\text { terminal }\end{array}$ & $\begin{array}{l}\text { Aproximándose al final de la vida. } \\
\text { Aplica para personas con una } \\
\text { expectativa de vida }<6 \text { meses que no } \\
\text { están evidentemente frágiles. }\end{array}$ \\
\hline
\end{tabular}

Puntaje 0-4: No frágil. 5-9: Frágil.

evaluar la relación entre la fragilidad y los desenlaces en pacientes llevados a tal procedimiento; se aplicó la escala de riesgo de $\mathrm{PCl}$ de Nueva York y la escala del Canadian Study of Health and Aging Clinical Frailty (tabla 4). El 11\% se clasificaron como frágiles, ninguno tuvo un puntaje mayor a 7 , el promedio fue de $3 \pm 1.3$. Los pacientes frágiles tuvieron una mayor predicción de la mortalidad por la escala de Nueva York, mayor intervalo de tiempo entre ingreso y coronariografía $(2.9 \pm 5.6$ días vs. $1.7 \pm 3.1$ días, $p<0.001)$, y mayor tiempo de hospitalización después de la revascularización $(14.1 \pm 26.7$ días vs. $3.5 \pm 8.8$ días, $p<0.001)$. La 
Tabla 5 Puntaje de Green ${ }^{17}$ (Escala 0-12)

\begin{tabular}{lll}
\hline Albúmina & $\leq 3.49$ & 3 \\
sérica (g/dl) & $3.5-3.69$ & 2 \\
& $3.7-3.99$ & 1 \\
& $\geq 4$ & 0 \\
Actividad física & Encuesta de & 0 =independiente \\
& Katz* para las & 3 = requiere \\
& actividades & asistencia para \\
& de la vida & cualquiera \\
& diaria & de las 6 \\
& $(6$ actividades & actividades \\
& en total) & \\
Velocidad de la & $\leq 0.57$ & 3 \\
marcha & $0.58-0.67$ & 2 \\
(4.57 m/tiempo & $0.68-0.89$ & 1 \\
para recorrer & $\geq 0.9$ & 0 \\
esta & & \\
distancia en & & \\
segundos) & & \\
Fuerza de & Mujeres & \\
presión & Hombres & \\
manual en Kg & $\leq 7.2 \leq 18.9$ & 2 \\
por & $7.3-11.3$ & 1 \\
dinamómetro & $19-25.6$ & 0 \\
& $11.4-15.6$ & \\
& $25.7-30.5$ & $15.7 \geq 30.6$ \\
\hline
\end{tabular}

*El índice de Katz es una escala sencilla que consta de seis elementos y evalúa las actividades básicas de la vida diaria proporcionando un índice de autonomía-dependencia en un breve tiempo de administración. Su carácter jerárquico permite evaluar el estado funcional global de forma ordenada, comparar individuos y grupos y detectar cambios a lo largo del tiempo.

mortalidad a 30 días fue casi 5 veces superior en pacientes frágiles frente a los no frágiles (HR 4.8, IC 95\% 1.4-16.3, $\mathrm{p}=0.01$ ), y la mortalidad a 1 año fue 6 veces mayor (HR 5.9, IC 95\% 2.5-13.8; $p<0.001$ ). En el análisis multivariado, la fragilidad fue un factor de riesgo independiente para la mortalidad a 30 días y tanto la fragilidad como la edad fueron factores de riesgo independientes de la mortalidad a 1 año.

Sanchis y Col., en su estudio sobre fragilidad y síndromes geriátricos en estratificación cardiaca de mayores de 65 años, concluyeron que la fragilidad medida a través del puntaje de Green (tabla 5), fue el único predictor independiente de mortalidad (HR 1.25, IC 95\% 1.15-1.36, p=0.001), y la muerte/infarto de miocardio a 30 días (HR 1.16, IC 95\% 1.09-1.24, $p=0.001)$. Un puntaje de Green $\geq 5$ fue el predictor de la mortalidad más fuerte, incluso sobre el puntaje de GRACE $^{17,18}$.

\section{Conclusiones}

La toma de decisiones clínicas en la población de adultos mayores, resulta retadora pues se conoce poco sobre la estrategia de manejo más apropiada para este grupo poblacional, dada su exclusión de los estudios y la presencia de múltiples comorbilidades. No obstante, considerando el progresivo incremento de la expectativa de vida y la cantidad de pacientes ancianos se han propuesto buscar respuesta a tales interrogantes. A la hora de estratificar el riesgo coronario se han planteado múltiples escalas específicas para esta población, muchas de las cuales requieren de validaciones para ser extrapoladas en la práctica clínica; sin embargo, ya ofrecen valiosas herramientas que permiten un acercamiento más adecuado y una toma de decisiones centrada en los adultos mayores. Se requieren más estudios multicéntricos que tengan aplicación y representación de poblaciones en nuestro medio, para lograr extrapolar estas escalas.

\section{Responsabilidades éticas}

Protección de personas y animales. Los autores declaran que para esta investigación no se han realizado experimentos en seres humanos ni en animales.

Confidencialidad de los datos. Los autores declaran que en este artículo no aparecen datos de pacientes.

Derecho a la privacidad y consentimiento informado. Los autores declaran que en este artículo no aparecen datos de pacientes.

\section{Conflicto de intereses}

Los autores del presente artículo no declaran ningún conflicto de intereses.

\section{Bibliografía}

1. Toledo-Franco LM. Identificar la fragilidad en pacientes con enfermedad cardiovascular: ¿opción u obligación? Rev Colomb Cardiol. 2015;22:205-6.

2. Fried LP, Tangen CM, Walston J, Newman AB, Hirsch C, Gottdiener J, et al. Frailty in older adults: evidence for a phenotype. J Gerontol A Biol Sci Med Sci. 2001;56:M146-56.

3. Saunderson CED, Brogan RA, Simms AD, Sutton G, Batin PD, Gale CP. Acute coronary syndrome management in older adults: guidelines, temporal changes and challenges. Age Ageing. Julio de. 2014; 43:450-5.

4. Alabas OA, Allan V, McLenachan JM, Feltbower R, Gale CP. Agedependent improvements in survival after hospitalisation with acute myocardial infarction: an analysis of the Myocardial Ischemia National Audit Project (MINAP). Age Ageing. Noviembre de. 2014;43:779-85.

5. Yan AT, Yan RT, Huynh T, Casanova A, Raimondo FE, Fitchett $\mathrm{DH}$, et al. Understanding physicians' risk stratification of acute coronary syndromes: insights from the Canadian ACS 2 Registry. Arch Intern Med. 2009;169:372-8.

6. Roe MT, Chen AY, Thomas L, Wang TY, Alexander KP, Hammill BG, et al. Predicting long-term mortality in older patients after nonST-segment elevation myocardial infarction: the CRUSADE longterm mortality model and risk score. Am Heart J. 2011;162:87583.e1.

7. Angeli F, Cavallini C, Verdecchia P, Morici N, Del Pinto M, Petronio AS, et al. A risk score for predicting 1-year mortality in patients $\geq 75$ years of age presenting with non-ST-elevation acute coronary syndrome. Am J Cardiol. 2015;116:208-13. 
8. Moretti C, Quadri G, D’Ascenzo F, Bertaina M, Giusto F, Marra S, et al. THE STORM (acute coronary Syndrome in patients end Of life and Risk asses Ment) study. Emerg Med J EMJ. 2015.

9. Maraschini A, D’Errigo P, Casali G, Rosato S, Badoni G, Seccareccia F. Risk stratification models in elderly patients: recalibrating or remodeling? Acta Cardiol. febrero de. 2013;68:11-8.

10. Hanon O, Baixas C, Friocourt P, Carrié D, Emeriau J-P, Galinier $M$, et al. Consensus of the French Society of Gerontology and Geriatrics and the French Society of Cardiology for the management of coronary artery disease in older adults. Arch Cardiovasc Dis. 2009;102:829-45.

11. Folstein MF, Folstein SE, McHugh PR. «Mini-mental state»A practical method for grading the cognitive state of patients for the clinician. J Psychiatr Res. Noviembre de. 1975;12:189-98.

12. Derouesne C, Poitreneau J, Hugonot L, Kalafat M, Dubois B, Laurent B. [Mini-Mental State Examination:a useful method for the evaluation of the cognitive status of patients by the clinician Consensual French version]. Presse Médicale Paris Fr 1983. 12 de junio de. 1999;28:1141-8.

13. Barberger-Gateau P, Dartigues JF, Letenneur L. Four Instrumental Activities of Daily Living Score as a predictor of one-year incident dementia. Age Ageing. Noviembre de. 1993;22:457-63.
14. Singh M, Alexander K, Roger VL, Rihal CS, Whitson HE, Lerman $A$, et al. Frailty and its potential relevance to cardiovascular care. Mayo Clin Proc. 2008;83:1146-53.

15. Murali-Krishnan R, lqbal J, Rowe R, Hatem E, Parviz Y, Richardson J, et al. Impact of frailty on outcomes after percutaneous coronary intervention: a prospective cohort study. Open Heart. 2015;2:e000294.

16. Sanchis J, Bonanad C, Ruiz V, Fernández J, García-Blas S, Mainar L, et al. Frailty and other geriatric conditions for risk stratification of older patients with acute coronary syndrome. Am Heart J. 2014; 168:784-91.

17. Green P, Woglom AE, Genereux P, Daneault B, Paradis J-M, Schnell $S$, et al. The impact of frailty status on survival after transcatheter aortic valve replacement in older adults with severe aortic stenosis: a single-center experience. JACC Cardiovasc Interv. 2012;5:974-81.

18. Sanchis J, Bonanad C, Ruiz V, Fernández J, García-Blas S, Mainar $\mathrm{L}$, et al. Frailty and other geriatric conditions for risk stratification of older patients with acute coronary syndrome. Am Heart J. 2014;168:784, 91.e2. 